\title{
On Bundling and Entry Deterrence
}

\author{
Andrea Greppi ${ }^{1}$. Domenico Menicucci ${ }^{2}$ (])
}

Published online: 27 August 2020

(c) The Author(s) 2020

\begin{abstract}
A multiproduct incumbent firm faces the threat of entry from another multiproduct (generalist) firm or from single-product (specialist) firms. Assuming that the incumbent offers higher quality products than its rivals, we inquire whether the possibility of bundling by the incumbent is more effective in deterring entry in one setting or the other, and explore how the quality difference affects the comparison. For instance, for relatively high-quality differences the generalist is more vulnerable to bundling than are the specialists; but bundling is a credible action for the incumbent more often against specialists than against the generalist.
\end{abstract}

Keywords Competitive bundling $\cdot$ Entry deterrence $\cdot$ Vertical differentiation

JEL Classification D43 $\cdot$ L13 $\cdot$ L41

\section{Introduction}

At least since Whinston (1990) it is known that an incumbent multiproduct firm may use bundling in order to reduce an entrant's profit-possibly below the entry costand thus build an entry barrier. This paper investigates how bundling is effective to deter entry, depending on whether the incumbent faces a single multiproduct rival or multiple single product rivals.

In the proposed merger between GE and Honeywell, one argument of the European Commission against the merger was that the merged firm would have had more than half of market share in the markets for airplane engines and for avionics, and

Domenico Menicucci

domenico.menicucci@unifi.it

Andrea Greppi

andrea.greppi2@unibo.it

1 Dipartimento di Scienze Economiche, Università degli Studi di Bologna, Piazza Scaravilli 2, 40126 Bologna, BO, Italy

2 Dipartimento di Scienze per l'Economia e l'Impresa, Università degli Studi di Firenze, Via delle Pandette 32, 50127 Florence, FI, Italy 
could have used bundling to induce its rivals' exit (see Nalebuff 2002). ${ }^{1}$ The merged entity would have competed with single-product firms, and we inquire whether a multiproduct opponent could better endure the effect of bundling; this also reveals whether single-product firms gain from merging.

Choi (2008) addresses the same question, relative to mixed bundling, in a framework with symmetric firms that he uses to analyze the effect of the proposed merger between GE and Honeywell. Conversely, we assume vertical differentiation in the sense that the incumbent offers products with higher quality than its rivals' products. ${ }^{2}$ We consider two games that differ because in one the incumbent faces an entry threat from a "generalist" multiproduct firm; in the other the entry threat comes from two "specialist" single product firms. After its rival(s) entry decision(s), the incumbent chooses whether to bundle or not its products; then price competition occurs.

We compare the two games and determine when, as a function of the extent of vertical differentiation, the incumbent's ability to bundle is more effective in deterring entry. About this, we notice that competition under bundling is fiercer, and less profitable for each firm, when the incumbent faces a generalist than when it faces specialists. Precisely, each specialist has a smaller incentive to cut its own price as it does not capture the full benefit from the price cut: the Cournot complement effect; hence specialists are less aggressive than is the generalist. This benefits the incumbent, and also induces it to be less aggressive (since prices are strategic complements); this makes the specialists' total profit higher than the generalist's profit.

Therefore, upon entry, the incumbent wants to bundle more often when it faces specialists; but when the incumbent bundles against a generalist, the reduction in the profit of the generalist is greater than the reduction in the profits of specialists. We show that as a consequence, for small-to-intermediate vertical differentiation, the incumbent's ability to bundle is a better entry deterrent against specialists because against a generalist, bundling reduces also the incumbent's profit, hence it is not credible. $^{3}$ Conversely, for intermediate-to-large vertical differentiation, bundling is a better foreclosure instrument against a generalist because it is profitable for the incumbent and reduces the generalist's profit more than the specialists' total profits.

The paper is organized as follows: The next section introduces the model, and Sect. 3 (Sect. 4) examines the game in which the incumbent faces a generalist (faces specialists). Section 5 compares the two games, and Sect. 6 concludes. The study

\footnotetext{
1 Our analysis is presented in terms of entry decisions; but similar arguments apply to the exit incentives for firms that are active in the market, and that incur a fixed cost of operation that can be saved by exiting.

2 This is consistent with the fact that before the proposed merger, GE (Honeywell) had more than 50 percent of market share in the market of aircraft engines (of avionics); see Nalebuff (2002).

3 In the baseline model of Whinston (1990), if entry occurs then bundling reduces also the incumbent's profit; hence a commitment by the incumbent is needed for bundling to deter entry. Also Choi and Stefanadis (2001) and Carlton and Waldman (2002) study bundling and entry deterrence when the incumbent can commit to bundling. Conversely, in our paper the incumbent has no ability to commit, as in Nalebuff (2004) and Peitz (2008).
} 
of some price competition subgames (Lemmas 1,4) relies on Hurkens et al. (2019) (HJM henceforth). The other results in this paper are original.

\section{The Setting}

We consider, and later compare, two competition settings. In one of them a multiproduct firm $A$, which offers products $A 1$ and $A 2$, is already active and faces the threat of entry of two single-product firms $B 1$ and $B 2$ such that firm $B j$ offers only product $B j$, for $j=1,2$. Each firm $B j$ incurs a cost $k>0$ if it decides to enter. In the other competition setting, firms $B 1$ and $B 2$ are merged into a single entity$B$-which offers products $B 1$ and $B 2$. Firm $A$ faces the threat of entry of firm $B$, for which the entry cost is $2 k$. Firms are Bertrand competitors in the sense that each firm chooses the price(s) at which it offers its product(s); but products of different firms are differentiated, hence the Bertrand paradox does not apply here.

After its rival(s) entry decisions(s), firm $A$ decides whether to offer its products $A 1, A 2$ separately or as a bundle - in the latter case, each consumer either buys $A 1$ and $A 2$ or buys no product from $A$ - that is, $A$ chooses separate sales or joint sales (i.e., bundling). ${ }^{4}$ Then firm $A$ competes with its active rival(s), or acts as a monopolist if no rival entered. We use $\Gamma^{s}$ to denote the game in which $A$ faces specialist firms $B 1, B 2 ; \Gamma^{g}$ denotes the game in which $A$ faces the generalist firm $B$. One way to interpret the comparison between $\Gamma^{g}$ and $\Gamma^{s}$ is to assume that $A^{\prime}$ s rival wants to maximize profit and can costlessly split into two firms if it is initially a generalist, can costlessly merge if it is initially split in two firms.

In either setting, each consumer has unit demand for good $j$, for $j=1,2$. Moreover, the firms are vertically differentiated in the sense that product $A j$ has a higher quality than product $B j$; the former yields to consumers a higher utility than does $B j$, for $j=1,2$. Precisely, each consumer has the same monetary gross utility $v_{i j}$ from consuming product $i j$, for $i=A, B, j=1,2$, such that $v_{B 1}=v_{B 2}=v>0$ and $v_{A 1}=v_{A 2}=v+\alpha$ with $\alpha>0$. Thus, $\alpha$ is the utility difference between products $A j$ and $B j$ and represents a measure of vertical differentiation between firms.

The firms are also horizontally differentiated in a standard Hotelling fashion: Relative to each good $j$, consumers are uniformly distributed over the interval $[0,1]$ (and independently across goods); firm $A$ is located at 0 ; and firm $B$ (or firm $B j$ ) is located at 1 . A consumer with location $x_{j} \in[0,1]$ for good $j$ incurs a transportation $\operatorname{cost} t x_{j}\left(t\left(1-x_{j}\right)\right)$ if he buys product $A j$ (product $B j$ ), with $t>0$. This consumer's net utility from buying (for instance) product $A j$ is $v+\alpha-t x_{j}$ minus his payment to firm $A$. Utility is additive over the two goods.

For each firm $i$, let $c$ denote the marginal production cost for product $i j$ (for $j=1,2)$. We assume that $c$ is larger than $t$ : If a consumer buys the bundle of $A$, then he will not also buy a single product $B j$ even though $B j$ yields a higher utility than $A j$

\footnotetext{
4 We rule out mixed bundling, in which a multiproduct firm offers both its products separately and the bundle. We conjecture that mixed bundling would not change our results qualitatively. See Choi (2008) for an analysis of mixed bundling in a different setting and with symmetric firms.
} 
(including the transportation costs), because the utility difference is smaller than $t$, the price of $B j$ is no less than $c$, and $c>t$. Then, since marginal costs have an additive effect on prices, without loss of generality we simplify the notation by setting $c=0$ and interpret prices as profit margins.

With regard to $v, \alpha, t$ we suppose that $v \geq 3 t>\alpha$. The inequality $v \geq 3 t$ implies that when both products $A j$ and $B j$ are offered, for $j=1,2$ - when each rival of $A$ enters-both markets are fully covered in equilibrium: Competition among firms leads to equilibrium prices such that each consumer buys one good 1 (product $A 1$ or $B 1$ ) and one good 2 (product $A 2$ or $B 2$ ). Hence, in this case we can view goods 1 and 2 as complements such that in order to obtain a positive utility, a consumer needs one unit of good 1 and one unit of good 2.

The property of fully covered markets does not hold if product $B 1$ and/or $B 2$ is not offered. For instance, if firm $A$ is a monopolist for both goods, then $A$ may want to charge prices such that not all consumers buy $A 1$ and $A 2$, as it is known from standard monopoly theory: see Sect. 3.1.2. This is socially inefficient because each consumer values one unit of good $j$ more than its production cost, for $j=1,2$; thus a social welfare loss (a deadweight loss) results. ${ }^{5}$

The inequality $\alpha<3 t$ implies that each active firm has a positive profit (gross of the entry cost) both for the case of competition under separate sales and for competition under bundling. ${ }^{6}$

In the rest of the paper, we derive our results in terms of $\alpha \in(0,3 t)$, the key parameter of our analysis, that reflects how strong vertical differentiation (measured by $\alpha$ ) is with respect to horizontal differentiation (measured by $t$ ). In particular, $\alpha$ affects not only price competition between firms, ${ }^{7}$ but also firms' preferences between competition under separate sales or under joint sales, which eventually affects the entry decision(s) of firm $B$ (of firms $B 1, B 2$ ).

\section{Competition Between Firm $A$ and Firm B: Game $\Gamma^{g}$}

In game $\Gamma^{g}$, the players are firm $A$ and firm $B$ and the timing is as follows:

- Stage one: Firm $B$ chooses between entering and not entering, and $A$ observes $B$ 's decision.

- Stage two: After $B$ 's entry decision, $A$ decides whether to offer its products separately (separate sales: "SS"), or in a bundle (joint sales: "JS").

\footnotetext{
${ }^{5}$ Likewise, if in $\Gamma^{s}$ only firm $B 1$ enters and firm $A$ bundles, then each consumer that buys product $B 1$ does not consume any good 2 (neither product $A 2$ nor $B 2$ ), which is socially inefficient: see Sect. 4.1.2.

${ }^{6}$ Both the inequalities $v \geq 3 t$ and $\alpha<3 t$ mainly simplify the exposition, and relaxing them does not yield additional insights. In Sect. 3.1.1 we illustrate the consequences of allowing $\alpha \geq 3 t$. With regard to $v \geq 3 t$, a weaker inequality suffices to generate the result of fully covered markets when all products are offered. However, $v \geq 3 t$ simplifies our presentation in Sect. 4.3 and in Sect. 5.

7 As is intuitive, a higher $\alpha$ favors firm $A$ and harms its rival(s).
} 
- Stage three: If firm $B$ has not entered, then $A$ acts as a monopolist. If $B$ has entered, then $A$ and $B$ compete under SS or under JS as determined by $A$ at stage two.

Notice that if $B$ has entered and $A$ has chosen SS, then at stage three firm $A(B)$ sets the prices $p_{A 1}, p_{A 2}\left(p_{B 1}, p_{B 2}\right)$ for its single products and each consumer buys one good 1 and one good 2 (precisely, this is the case at the equilibrium prices). In particular, each consumer may "mix and match", that is buy one product from firm $A$ and one from firm $B$, selecting for instance products $A 1, B 2$ and paying a total of $p_{A 1}+p_{B 2}$. Conversely, if $A$ has chosen JS then competition occurs between bundles because each consumer either buys $A$ 's bundle, or buys the products $B 1, B 2$, which therefore are bundled in practice. In this case firm $A(B)$ sets the price $P_{A}\left(P_{B}\right)$ for the bundle of its products. ${ }^{8}$

In this section we use backward induction to identify the subgame perfect Nash equilibria (SPNE) of $\Gamma^{g}$ : For each terminal subgame of $\Gamma^{g}$ (a subgame that starts at stage three) we identify the unique equilibrium and the equilibrium profits. These are used to deduce $A$ 's stage two choice about bundling or not, and ultimately $B$ 's entry decision at stage one.

\subsection{Stage Three in $\Gamma^{g}$}

\subsubsection{Subgames Such That Firm B Has Entered}

Given that firm $B$ has entered, competition at stage three is affected by $A$ 's choice at stage two.

If competition occurs under SS, each market can be analyzed in isolation. In market $j$, a consumer located at $x_{j}$ buys product $A j$ if and only if his utility with $A j$ is higher than with $B j$ : if and only if $v+\alpha-t x_{j}-p_{A j} \geq v-t\left(1-x_{j}\right)-p_{B j}$, which reduces to

$$
x_{j} \leq \frac{1}{2 t}\left(\alpha+t+p_{B j}-p_{A j}\right) \equiv x_{j}^{m}
$$

in which $x_{j}^{m}$ is the location of the marginal consumer in market $j$. Moreover, $F(f)$ denotes the c.d.f. (the density) for the uniform distribution with support $[0,1]$; hence

$$
F(x)=x \quad f(x)=1 \quad \text { for each } x \in[0,1] .
$$

Therefore the demand for product $A j$ is $F\left(x_{j}^{m}\right) ; p_{A j} F\left(x_{j}^{m}\right)$ is $A$ 's profit in market $j$; and $p_{B j}\left(1-F\left(x_{j}^{m}\right)\right)$ is $B$ 's profit in market $j$ gross of the entry cost.

\footnotetext{
${ }^{8}$ We assume that only firm $A$ decides whether to bundle or not because allowing also $B$ to bundle would not change our results. Lemmas 1(3-4) below establish that if $B$ wants to bundle, then $A$ also wants to bundle. Hence competition occurs under JS if and only if firm $A$ prefers it to competition under SS.
} 
If competition occurs under JS, a consumer located at $\left(x_{1}, x_{2}\right)$ buys $A$ 's bundle if and only if $2 v+2 \alpha-t\left(x_{1}+x_{2}\right)-P_{A} \geq 2 v-t\left(1-x_{1}+1-x_{2}\right)-P_{B}$, which is equivalent to

$$
\bar{x} \leq \frac{1}{2 t}\left(\alpha+t+p_{B}-p_{A}\right) \equiv \bar{x}^{g m},
$$

in which: $\bar{x}=\left(x_{1}+x_{2}\right) / 2$ is the consumer's average location; $p_{A}=P_{A} / 2$ ( $\left.p_{B}=P_{B} / 2\right)$ is the average price of the products in $A$ 's bundle (in $B$ 's bundle); and $\bar{x}^{g m}$ is the average location of the marginal consumer.

Comparing (1) and (3) shows that competition under JS is analogous to competition under SS in the market for a single product, except that the distribution of the average location is not uniform: The c.d.f. $\bar{F}$ and the density $\bar{f}$ of the average location are

$$
\bar{F}(x)=\left\{\begin{array}{cl}
2 x^{2} & \text { if } 0 \leq x \leq \frac{1}{2} \\
1-2(1-x)^{2} & \text { if } \frac{1}{2}<x \leq 1
\end{array} \quad \bar{f}(x)=\left\{\begin{array}{cc}
4 x & \text { if } 0 \leq x \leq \frac{1}{2} \\
4(1-x) & \text { if } \frac{1}{2}<x \leq 1
\end{array} .\right.\right.
$$

Hence, the demand for the bundle of $A$ is $\bar{F}\left(\bar{x}^{g m}\right) ; P_{A} \bar{F}\left(\bar{x}^{g m}\right)$ is the profit of $A$; and $P_{B}\left(1-\bar{F}\left(\bar{x}^{g m}\right)\right)$ is the profit of $B$ gross of the entry cost. In the following, we denote with $\pi_{i}^{g}$ (with $\Pi_{i}^{g}$ ) the equilibrium profit—upon entry of $B$ - of firm $i$ in $\Gamma^{g}$ under SS (under JS), for $i=A, B$ :

Lemma 1 (HJM) In game $\Gamma^{g}$, suppose that firm B has entered. Then

(1) Under SS, the equilibrium prices and profits for firms $A$ and $B$ are:

$$
\begin{array}{cc}
p_{A 1}^{g}=p_{A 2}^{g}=t+\frac{1}{3} \alpha \equiv p_{A}^{*}, & p_{B 1}^{g}=p_{B 2}^{g}=t-\frac{1}{3} \alpha \equiv p_{B}^{*}: \\
\pi_{A}^{g}=\frac{(3 t+\alpha)^{2}}{9 t}, & \pi_{B}^{g}=\frac{(3 t-\alpha)^{2}}{9 t}
\end{array}
$$

(2) Under JS, the equilibrium prices and profits for firms A and B are:

$$
\begin{array}{lrr}
P_{A}^{g} & =\frac{5}{4}(\alpha-t)+\frac{3}{4} \beta^{g}, \quad P_{B}^{g}=\frac{1}{4}\left(t-\alpha+\beta^{g}\right) \quad \text { with } & \beta^{g}=\sqrt{(\alpha-t)^{2}+8 t^{2}} \\
\Pi_{A}^{g} & =\frac{\left(11 t^{2}+2 \alpha t-\alpha^{2}+(\alpha-t) \beta^{g}\right)^{2}}{32 t^{2}\left(t-\alpha+\beta^{g}\right)}, & \Pi_{B}^{g}=\frac{\left(t-\alpha+\beta^{g}\right)^{3}}{128 t^{2}}
\end{array}
$$

(3) If $\alpha<\alpha_{A}^{g}$, then $\pi_{A}^{g}>\Pi_{A}^{g}$; if $\alpha>\alpha_{A_{g}}^{g}$, then $\pi_{A_{g}}^{g}<\Pi_{A}^{g}$, with $\alpha_{A_{g}}^{g}=1.415 t$.

(4) If $\alpha<\alpha_{B}^{g}$, then $\pi_{B}^{g}>\Pi_{B}^{g}$; if $\alpha>\alpha_{B}^{g}$, then $\pi_{B}^{g}<\Pi_{B}^{g}$, with $\alpha_{B}^{g}=2.376$ t.

With respect to the analysis of game $\Gamma^{g}$, Lemma 1(3) is important as it determines the choice of firm $A$ at stage two between SS and JS, upon $B$ 's entry (see 
Lemma 3(1) below). Therefore, in the rest of this subsection we first provide some remarks about Lemma 1(1) and competition under SS, then we explain why JS leads to different prices and profits with respect to SS, and how $\alpha$ determines whether SS or JS is more profitable for firm $A$.

Lemma 1(1) identifies the equilibrium prices $p_{A}^{*}, p_{B}^{*}$ for the single products under SS. Notice that $p_{A}^{*}>p_{B}^{*}$, which is intuitive given the higher quality of $A$ 's products; but $p_{A}^{*}-p_{B}^{*}$ is smaller than the quality difference $\alpha$. We explain why by considering market 1: The demand for product $B 1$ is $D_{B 1}\left(p_{B 1}\right)=1-F\left(\left(\alpha+t+p_{B 1}-p_{A 1}\right) / 2 t\right)$, with elasticity $p_{B 1} D_{B 1}^{\prime}\left(p_{B 1}\right) / D_{B 1}\left(p_{B 1}\right)$. This must be -1 at $p_{B 1}=p_{B}^{*}$ in order for $B$ 's profit to be maximized; and if $p_{A}^{*}$ were equal to $p_{B}^{*}+\alpha$, then $D_{B 1}\left(p_{B}^{*}\right)=1 / 2$, $D_{B 1}^{\prime}\left(p_{B}^{*}\right)=-1 /(2 t)$; thus $p_{B}^{*} D_{B 1}^{\prime}\left(p_{B}^{*}\right) / D_{B 1}\left(p_{B}^{*}\right)=-1$ implies $p_{B}^{*}=t$. For product $A 1$, the elasticity is $p_{A 1} D_{A 1}^{\prime}\left(p_{A 1}\right) / D_{A 1}\left(p_{A 1}\right)$ and $p_{A}^{*}=p_{B}^{*}+\alpha, p_{B}^{*}=t$ imply $p_{A}^{*} D_{A 1}^{\prime}\left(p_{A}^{*}\right) / D_{A 1}\left(p_{A}^{*}\right)=-1-\alpha / t<-1$ : the demand for $A 1$ is elastic, and $A$ gains by reducing $p_{A 1}$ below $p_{B}^{*}+\alpha$, which is consistent with (5).

Using (1) and (5) we see that in each market the marginal consumer is located at $x^{*}=1 / 2+\alpha /(6 t)$, and $x^{*} \in(1 / 2,1)$ since $\alpha \in(0,3 t)$. A consumer who is located at $x_{j}$ for good $j$ buys $A j$ if $x_{j}<x^{*}$, or buys $B j$ if $x_{j}>x^{*}$. Hence, consumers with location smaller than $x^{*}$ for one good but greater than $x^{*}$ for the other good mix and match: They buy one good from firm $A$, and one good from firm $B$.

As we mentioned in Sect. 2, when $\alpha<3 t$, each firm has a positive profit under SS. If instead $\alpha \geq 3 t$, then in each market $j$ the equilibrium prices are $p_{A j}^{*}=\alpha-t$, $p_{B j}^{*}=0$; each consumer buys product $A j$, and $B$ 's profit is zero. The inequality $\alpha \geq 3 t$ is necessary for this equilibrium as it guarantees that $A$ has no incentive to increase $p_{A j}$ above $\alpha-t$ : When $\alpha \geq 3 t, p_{A j}^{*}$ is large enough that an increase in $p_{A j}$ generates a profit decrease for $A$ from consumers lost that is greater than the profit increase from the consumers that keep buying product $A j$.

With respect to competition under JS: Lemma 1(2) shows that $P_{A}^{g} \neq 2 p_{A}^{*}$ and $P_{B}^{g} \neq 2 p_{B}^{*}$ : The equilibrium price for each bundle under JS is different from the sum of the equilibrium prices under SS for the products in the bundle. Moreover, also the profits under JS are different from the profits under SS. This occurs because under SS the demand functions are determined by $F$ in (2), the c.d.f. of consumers' locations for a single good; but under JS $F$ is replaced by $\bar{F}$ in (4), the c.d.f. of the consumers' average locations. This modifies the demand elasticities and demand sizes, which affect the firms' pricing incentives and profits.

In order to make clearer the difference between $F$ and $\bar{F}$, Fig. 1 represents $f, \bar{f}$ and $F, \bar{F}$ from (2) and (4) (please see next page).

Both $f$ and $\bar{f}$ are symmetric around $1 / 2$ (the mean), but $\bar{f}$ is more peaked than $f$ around $1 / 2$, that is $\bar{f}$ puts more weight around $1 / 2$ than does $f$, but puts less weight near 0 and near 1 . This implies (see Fig. 1b)

$$
\bar{F}(x)>F(x) \quad \text { for each } \quad x \in\left(\frac{1}{2}, 1\right) .
$$

In order to examine the effect of JS, we first suppose that $\left(P_{A}, P_{B}\right)=\left(2 p_{A}^{*}, 2 p_{B}^{*}\right)$, that is the price for each bundle is the sum of the prices under SS of the products in the bundle. Under JS, the demand function for the bundle of $A$ (of $B$ ) is $\bar{F}\left(\bar{x}^{g m}\right)$ (is 


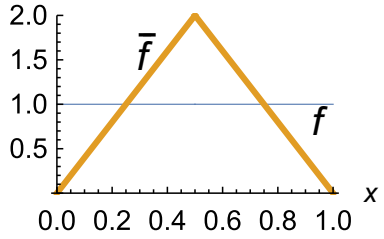

(a)

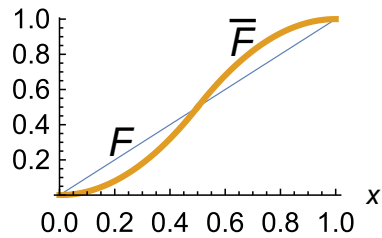

(b)

Fig. 1 a Density of the uniform distribution (thin) and of the average (thick). b c.d.f. of the uniform distribution (thin) and of the average (thick)

$\left.1-\bar{F}\left(\bar{x}^{g m}\right)\right)$; and given $\left(P_{A}, P_{B}\right)=\left(2 p_{A}^{*}, 2 p_{B}^{*}\right)$ we have that $\bar{x}^{g m}$ is equal to $x^{*}$ : the location of the equilibrium marginal consumer (in both markets) under SS. Therefore, firm $A$ 's demand changes from $F\left(x^{*}\right)$ for each product of $A$ to $\bar{F}\left(x^{*}\right)$ for $A$ 's bundle, and $x^{*} \in(1 / 2,1)$ plus (7) imply $\bar{F}\left(x^{*}\right)>F\left(x^{*}\right)$. Hence, JS with unchanged unit prices increases the demand for $A$, and reduces the demand for $B$.

This is the demand size effect, according to the terminology in HJM. It arises because (1) under SS, product $A j$ is purchased by consumers who are located in $\left[0, x^{*}\right]$ relative to good $j ;(2)$ under JS with $\left(P_{A}, P_{B}\right)=\left(2 p_{A}^{*}, 2 p_{B}^{*}\right), A$ 's bundle is purchased by consumers with average location in $\left[0, x^{*}\right]$; and (3) $A$ 's quality advantage implies $x^{*}>1 / 2$ and there are more consumers in $\left[0, x^{*}\right]$ under the c.d.f. $\bar{F}$ than under $F: \bar{F}\left(x^{*}\right)>F\left(x^{*}\right)$, because $\bar{f}$ puts more weight around $1 / 2$ than $f$. Thus JS increases $A$ 's demand because it makes the distribution of consumers' locations more concentrated near $1 / 2$, and consumers close to $1 / 2$ buy from $A$ because of $A$ 's quality advantage. But notice that this effect is weak if $x^{*}$ is close to $1 / 2$ or close to 1 as then $\bar{F}\left(x^{*}\right)-F\left(x^{*}\right)$ is small.

Moving to JS also generates a demand elasticity effect that induces firm $i$ to set the price $P_{i}$ of its bundle different from $2 p_{i}^{*}$, for $i=A, B$ : The demand for $A$ 's bundle is $\bar{F}\left(\bar{x}^{g m}\right)$ and its elasticity with respect to $P_{A}$ at $\left(P_{A}, P_{B}\right)=\left(2 p_{A}^{*}, 2 p_{B}^{*}\right)$ is $-\left(p_{A}^{*} / 2 t\right)\left(\bar{f}\left(x^{*}\right) / \bar{F}\left(x^{*}\right)\right)$, whereas under SS the elasticity of $F\left(x_{j}^{m}\right)$ (the demand for product $A j$ ) with respect to $p_{A j}$ at $\left(p_{A j}, p_{B j}\right)=\left(p_{A}^{*}, p_{B}^{*}\right)$ is $-\left(p_{A}^{*} / 2 t\right)\left(f\left(x^{*}\right) / F\left(x^{*}\right)\right)$, equal to -1 since $p_{A}^{*}$ is a best reply for $A$ to $p_{B}=p_{B}^{*}$. In the following we identify the demand elasticity effect for firm $A$ by comparing $-\left(p_{A}^{*} / 2 t\right)\left(\bar{f}\left(x^{*}\right) / \bar{F}\left(x^{*}\right)\right)$ with $-\left(p_{A}^{*} / 2 t\right)\left(f\left(x^{*}\right) / F\left(x^{*}\right)\right)$.

When $\alpha$ is about zero, we have that $x^{*}$ is about 1/2; thus (see (2), (4) or Fig. 1) $\bar{F}\left(x^{*}\right)$ and $F\left(x^{*}\right)$ are both close to $1 / 2$ but $\bar{f}\left(x^{*}\right)$ is about twice as large as $f\left(x^{*}\right)$. Hence $-\left(p_{A}^{*} / 2 t\right)\left(\bar{f}\left(x^{*}\right) / \bar{F}\left(x^{*}\right)\right)<-\left(p_{A}^{*} / 2 t\right)\left(f\left(x^{*}\right) / F\left(x^{*}\right)\right)$ : $A^{\prime}$ 's demand is more elastic under JS than under SS, as under JS more consumers are located near the marginal consumer; this is a consequence of the fact that $\bar{f}$ puts more weight around $1 / 2$ than does $f$. Conversely, when $\alpha$ is large, close to $3 t, x^{*}$ is about 1 , and (see (2), (4) or Fig. 1) $\bar{F}\left(x^{*}\right), F\left(x^{*}\right)$ are both about 1 but $\bar{f}\left(x^{*}\right)$ is close to 0 , smaller than $f\left(x^{*}\right)=1$. Thus $-\left(p_{A}^{*} / 2 t\right)\left(\bar{f}\left(x^{*}\right) / \bar{F}\left(x^{*}\right)\right)>-\left(p_{A}^{*} / 2 t\right)\left(f\left(x^{*}\right) / F\left(x^{*}\right)\right)$, that is under JS firm $A$ 's demand is less elastic because fewer consumers are located near the marginal consumer, as $\bar{f}$ puts less weight near 1 than does $f$. 
For the demand elasticity effect for firm $B$ we notice that $1-\bar{F}\left(\bar{x}^{g m}\right)$ is the demand for $B$ 's bundle and its elasticity with respect to $P_{B}$ at $\left(P_{A}, P_{B}\right)=\left(2 p_{A}^{*}, 2 p_{B}^{*}\right)$ is $-\left(p_{B}^{*} / 2 t\right)\left(\bar{f}\left(x^{*}\right) /\left(1-\bar{F}\left(x^{*}\right)\right)\right)$. From (2), (4) we see that for each $x^{*} \in(1 / 2,1)$, this is smaller than -1 , the demand elasticity $-\left(p_{B}^{*} / 2 t\right)\left(f\left(x^{*}\right) /\left(1-F\left(x^{*}\right)\right)\right)$ for each single product of $B$ under SS. This occurs because the demand size effect implies that $1-\bar{F}\left(x^{*}\right)<1-F\left(x^{*}\right)$, which makes $-\left(p_{B}^{*} / 2 t\right)\left(\bar{f}\left(x^{*}\right) /\left(1-\bar{F}\left(x^{*}\right)\right)\right)$ smaller than $-\left(p_{B}^{*} / 2 t\right)\left(f\left(x^{*}\right) /\left(1-F\left(x^{*}\right)\right)\right)$ even when $\bar{f}\left(x^{*}\right)<f\left(x^{*}\right)$. Thus the demand elasticity effect makes $B$ willing to reduce $P_{B}$ below $2 p_{B}^{*}$.

Summarizing: For $\alpha$ close to 0 the demand size effect is weak, and the demand elasticity effect induces both firms to reduce prices under JS. Then a more intense competition results, which makes both firms worse off under JS than under SS. In essence, if vertical differentiation is small, then JS intensifies competition and reduces profits because firms compete in a region with a high density of consumers (the marginal consumer $x^{*}$ is near $1 / 2$ as firms are almost symmetric when $\alpha$ is about zero, and $\left.\bar{f}\left(x^{*}\right)>f\left(x^{*}\right)\right)$, which provides incentives for aggressive pricing.

Conversely, for a large $\alpha$ the demand size effect is still weak, but now the demand for $A$ 's bundle is inelastic. This induces firm $A$ to increase $P_{A}$, which benefits firm $B$ because it softens the competition that $B$ faces. Moreover, the increase in $P_{A}$ above $2 p_{A}^{*}$ induces $B$ to increase $P_{B}$ since prices are strategic complements, even though $B$ 's demand is elastic at $\left(P_{A}, P_{B}\right)=\left(2 p_{A}^{*}, 2 p_{B}^{*}\right)$. Then less intense competition results, which makes $A$ and $B$ better off under JS. In essence, if vertical differentiation is large, then JS softens competition and increases profits because firms compete in a region with a low density of consumers (the marginal consumer $x^{*}$ is near 1 since $A$ 's quality advantage is large, and $\bar{f}\left(x^{*}\right) \simeq 0<f\left(x^{*}\right)=1$ ), which leads to less aggressive pricing.

Finally, for intermediate $\alpha$ the demand size effect is non-negligible (unlike in the extreme cases above), and it dominates the demand elasticity effect. As a result, $A$ is better off, and $B$ is worse off under JS than under SS: $\Pi_{A}^{g}>\pi_{A}^{g}$ and $\Pi_{B}^{g}<\pi_{B}^{g}$ for $\alpha \in\left(\alpha_{A}^{g}, \alpha_{B}^{g}\right){ }^{9}$

\subsubsection{Subgames Such That Firm B Has Not Entered}

If firm $B$ has not entered, then $A$ is a monopolist. Under SS, in market $j$ a consumer who is located at $x_{j}$ buys product $A j$ if and only if his utility from the purchase is non-negative: if $v+\alpha-t x_{j}-p_{A j} \geq 0$. Hence, the demand for product $A j$ is $F\left(\left(v+\alpha-p_{A j}\right) / t\right)$. Since $v \geq 3 t$, we find that the profit maximizing $p_{A j}$ is $v+\alpha-t$, the lowest possible consumer's valuation for $A j$; at this price, each consumer buys Aj.

\footnotetext{
${ }^{9}$ Since a high $\alpha$ implies that moving from SS to JS softens competition, we may investigate the incentive of firm $B$ to reduce the quality of its own products, which increases $\alpha$, in order to induce $A$ to choose JS instead of SS at stage two: After $B$ has entered, its profit is $\pi_{B}^{g}$ if $\alpha<\alpha_{A}^{g}$, and is $\Pi_{B}^{g}$ if $\alpha>\alpha_{A}^{g}$-taking into account $A$ 's choice at stage two. Although $\pi_{B}^{g}$ and $\Pi_{B}^{g}$ are both decreasing in $\alpha$, if at $\alpha=\alpha_{A}^{g}$ the inequality $\Pi_{B}^{g}>\pi_{B}^{g}$ were satisfied, then in some cases it would be convenient for $B$ to suitably increase $\alpha$. However, Lemma 1(4) implies that $\Pi_{B}^{g}<\pi_{B}^{g}$ at $\alpha=\alpha_{A}^{g}$; thus no increase in $\alpha$ is profitable for $B$.
} 
Now suppose that $A$ has chosen JS at stage two. A consumer who is located at $\left(x_{1}, x_{2}\right)$ buys the bundle of $A$ if and only if $2 v+2 \alpha-t\left(x_{1}+x_{2}\right)-P_{A} \geq 0$ : if $\left(2 v+2 \alpha-P_{A}\right) /(2 t) \geq \bar{x}$, in which $\bar{x}=\left(x_{1}+x_{2}\right) / 2$. Hence, the demand for $A$ 's bundle is $\bar{F}\left(\left(2 v+2 \alpha-P_{A}\right) /(2 t)\right)$.

\section{Lemma 2}

(1) If firm A is a monopolist under SS, then the optimal price in market $j$ is $p_{A j}=v+\alpha-t$, for $j=1,2$, and A's total profit is $2(v+\alpha-t)$.

(2) If firm A is a monopolist under JS, then the optimal price for the bundle is slightly greater than $2(v+\alpha-t)$, and A's profit is greater than $2(v+\alpha-t)$.

About Lemma 2(2), notice that $P_{A}=2(v+\alpha-t)$ under JS is equivalent to $p_{A 1}=p_{A 2}=v+\alpha-t$ under SS, as it induces each consumer to buy $A$ 's bundle and makes $A$ 's profit equal to $2(v+\alpha-t)$, which is also $A$ 's maximal profit under SS. However, $A$ can do better by slightly increasing $P_{A}$ above $2(v+\alpha-t)$, as we explain below. ${ }^{10}$

Under monopoly by $A$, we can view the consumers' valuations for product $A 1$ as uniformly distributed over the interval $[\underline{v}, \bar{v}]$, with $\underline{v}=v+\alpha-t$ and $\bar{v}=v+\alpha,{ }^{11}$ and the consumers' valuations for product $\bar{A} 2$ as uniformly distributed over the same interval $[\underline{v}, \bar{v}]$. Under SS, consider $p_{A 1}=\underline{v}+\varepsilon$ with $\varepsilon>0$ and small. This $p_{A 1}$ is less profitable than $p_{A 1}=\underline{v}$ because it induces a consumer not to buy product $A 1$ if his value $v_{1}$ for $A 1$ is between $\underline{v}$ and $\underline{v}+\varepsilon$ : if $v_{1}$ is close to $\underline{v}$, independently of the consumer's value $v_{2}$ for product $A 2$. By Lemma 2(1), this profit decrease for firm $A$ exceeds the profit increase from consumers that buy $A 1$ after the price increase. A similar argument explains why $p_{A 2}=\underline{v}+\varepsilon$ is less profitable than $p_{A 2}=\underline{v}$.

Now consider JS and $P_{A}=2 \underline{v}+2 \varepsilon$ : a bundle price that is slightly above $2 \underline{v}$. Then a consumer with values $v_{1}, v_{2}$ does not buy the bundle if and only if $v_{1}+v_{2}<2 \underline{v}+2 \varepsilon$ : if and only if $v_{1}, v_{2}$ are both close to $\underline{v}$. Thus the demand reduction that follows the price increase is much smaller under JS than under SS because under JS it comes only from consumers with both values close to $\underline{v}$, whereas under SS it comes from each consumer with at least one value close to $\underline{v}$. Then the profit increase from the consumers who buy the bundle after the increase in $P_{A}$ (this is about equal to the profit increase under SS from consumers buying the product(s) after the increases in $\left.p_{A 1}, p_{A 2}\right)$ makes the price increase profitable under JSalthough it is not so under SS. ${ }^{12}$

\footnotetext{
10 This result can be seen as an application of the Exclusion Principle described in Armstrong (1996).

11 A consumer with valuation $v_{1}$ for product $A 1$ equal to $\underline{v}$ (equal to $\bar{v}$ ) is located at $x_{1}=1$ (at $x_{1}=0$ ).

12 This argument captures the essence of the difference between SS and JS; but a complete analysis should take into account that the demand reduction under SS is more often about a single product than about both products, whereas the demand reduction under JS is about the whole bundle: both products. However, this does not affect the conclusion that a small increase in $P_{A}$ above $2 \underline{v}$ under JS lowers demand much less than a small increase in $p_{A 1}, p_{A 2}$ above $\underline{v}$ under SS. This can be seen also by noticing that the demand elasticity for the bundle under JS is $-\left(P_{A} / 2 t\right)\left(\bar{f}\left(\left(2 v+2 \alpha-P_{A}\right) / 2 t\right) / \bar{F}\left(\left(2 v+2 \alpha-P_{A}\right) / 2 t\right)\right)$, which is zero at $P_{A}=2 v=2(v+\alpha-t)$ as $\bar{f}(1)=0$.
} 
The difference between SS and JS arises because under JS consumers are forced to buy the bundle or no product at all. This implies that a consumer does not buy the bundle after the increase in $P_{A}$ only if his values are both close to $\underline{v}$. Conversely, SS allows each consumer to buy a single product. Hence, after the increases in $p_{A 1}, p_{A 2}$, a consumer with $v_{1}$ that is close to $\underline{v}$ and $v_{2}$ that is not too small-say high for brevity-chooses to buy only product $\bar{A} 2$, and a similar remark holds if $v_{2}$ is close to $\underline{v}$ and $v_{1}$ is high. Therefore, the price increase(s) lead to different allocations such that the demand reduction is greater under SS. For instance, a consumer with low $v_{1}$ and high $v_{2}$ buys only A2 under SS, but buys the bundle under JS because of the high $v_{2}$.

\subsection{Stage Two in $\Gamma^{g}$}

At stage two, firm $A$ chooses between SS and JS. Such choice is determined by the profits $A$ expects to earn under SS and under JS, and by whether firm $B$ has entered or not. Lemma 1(3) shows that if firm $B$ has entered, then the comparison between $\pi_{A}^{g}$ and $\Pi_{A}^{g}$ depends on $\alpha$ (as we illustrated after Lemma 1), whereas Lemma 2 shows that this is not the case if $B$ has not entered. Using these results, we deduce $A$ 's choice at stage two.

Lemma 3 In game $\Gamma^{g}$, at stage two

(1) if firm $B$ has entered at stage one, then A's best action is SS if $\alpha<\alpha_{A}^{g}$, and is JS if $\alpha>\alpha_{A}^{g}$;

(2) if firm B has not entered, then A's best action is JS.

\subsection{Stage One in $\Gamma^{g}$}

Here we study the entry decision of firm $B$, which relies on anticipating the equilibrium play at the successive stages, described by Lemmas 1,3 . When $\alpha<\alpha_{A}^{g}$, firm $B$ knows that $A$ will play SS in case of entry; since $B$ 's entry cost is $2 k, B$ compares $\pi_{B}^{g}$ with $2 k$. When $\alpha>\alpha_{A}^{g}$, $A$ will choose JS if $B$ enters, hence $B$ compares $\Pi_{B}^{g}$ with $2 k$. This yields Proposition 1:

Proposition 1 In game $\Gamma^{g}$, the unique SPNE is such that

(1) when $\alpha<\alpha_{A}^{g}$, at stage one firm $B$ enters if and only if $k<\pi_{B}^{g} / 2$;

(2) when $\alpha_{A}^{g}<\alpha$, at stage one firm $B$ enters if and only if $k<\Pi_{B}^{g} / 2$;

the rest of the SPNE strategies (relative to stages two and three) is obtained from Lemmas 1-3.

Therefore $A$ 's ability to bundle does not deter $B$ 's entry if $\alpha<\alpha_{A}^{g}$, as it is not credible that $A$ bundles if $B$ enters. If instead $\alpha>\alpha_{A}^{g}$, then JS is credible but reduces $B$ 's profit only for $\alpha<\alpha_{B}^{g}$. Hence, $A$ 's ability to bundle helps to keep $B$ out only for $\alpha \in\left(\alpha_{A}^{g}, \alpha_{B}^{g}\right)$. 


\section{Competition Among Firm $A$ and Firms B1, B2: Game $\Gamma^{s}$}

In game $\Gamma^{s}$, the players are $A$ and the specialist firms $B 1, B 2$. The timing is as follows:

- Stage one: Firms $B 1$ and $B 2$ simultaneously and non-cooperatively decide whether to enter or not.

- Stage two: After observing the entry decisions of $B 1$ and $B 2$, firm $A$ chooses SS or JS.

- Stage three: If no specialist has entered, then $A$ acts as a monopolist. If $B 1$ and/ or $B 2$ has entered, then the active firms compete under SS or under JS, as determined by $A$.

In the event that $B 1, B 2$ have entered and $A$ has chosen SS, competition occurs essentially as in game $\Gamma^{g}$, except that firm $B 1$ (firm $B 2$ ) sets $p_{B 1}\left(p_{B 2}\right)$. If instead $A$ has chosen JS, then competition takes place between the bundle of $A$ and the bundle of products $B 1 \& B 2$; firm $A$ sets $P_{A}$, firms $B 1, B 2$ choose $P_{B 1}, P_{B 2}$ and $P_{B 1}+P_{B 2}$ is the price of the bundle $B 1 \& B 2$.

In particular, under JS the so-called Cournot complement effect applies: If firm $B 1$ reduces $P_{B 1}$, then the demand for bundle $B 1 \& B 2$ increases since the goods can be seen as complements, as we remarked in Sect. $2 .{ }^{13}$ However, firm $B 1$ does not take into account that a reduction in $P_{B 1}$ increases the profit of $B 2$ and chooses $P_{B 1}$ to maximize just its own profit. Conversely, an integrated firm $B$ takes this externality into account and charges a bundle price that is lower than the total price charged by $B 1, B 2$. As a consequence, under JS firm $A$ prefers to compete against $B 1, B 2$ rather than against $B$ because $B 1, B 2$ are less aggressive. Under SS, there is no such effect because there is no link between the sale of $B 1$ and the sale of $B 2$.

As for $\Gamma^{g}$, we apply backward induction to $\Gamma^{s}$ and begin with the study of terminal subgames.

\subsection{Stage Three in $\Gamma^{s}$}

\subsubsection{Subgames Such That Both B1 and B2 Have Entered}

When competition occurs under SS after both $B 1$ and $B 2$ have entered, the analysis of Sect. 3.1.1 still applies: There are no links between market 1 and market 2 , hence when $A$ faces the separate entities $B 1, B 2$, the equilibrium prices are still $p_{A}^{*}, p_{B}^{*}$ in (5) and the equilibrium profit of $B 1(B 2)$ is half the equilibrium profit of $B$ in $\Gamma^{g}$. In order to simplify notation for the rest of the paper, we define $\hat{\pi}=\pi_{B}^{g} / 2=(3 t-\alpha)^{2} /(18 t)$; therefore $\hat{\pi}$ is the profit of both $B 1$ and $B 2$ under SS. Using $\hat{\pi}$, Proposition 1(1) can be stated as follows: When $\alpha<\alpha_{A}^{g}$, the unique SPNE of $\Gamma^{g}$ is such that firm $B$ enters if and only if $k<\hat{\pi}$.

$\overline{13}$ This effect is pointed out by Denicolò (2000) and Nalebuff (2000) in related models. 
When competition occurs under JS, (3) shows that a consumer who is located at $\left(x_{1}, x_{2}\right)$ buys $A$ 's bundle if and only if his average location $\bar{x}$ satisfies

$$
\bar{x} \leq \frac{1}{2 t}\left(\alpha+t+\frac{1}{2} P_{B 1}+\frac{1}{2} P_{B 2}-\frac{1}{2} P_{A}\right) \equiv \bar{x}^{s m} .
$$

Hence, $\bar{x}^{s m}$ is the average location of the marginal consumer. The demand for the bundle of $A$ is $\bar{F}\left(\bar{x}^{s m}\right), A$ 's profit is $P_{A} \bar{F}\left(\bar{x}^{s m}\right)$, and the profits for firms $B 1, B 2$ (gross of the entry costs) are $P_{B 1}\left[1-\bar{F}\left(\bar{x}^{s m}\right)\right], P_{B 2}\left[1-\bar{F}\left(\bar{x}^{s m}\right)\right]$, respectively. We use $\pi_{i}^{s}\left(\Pi_{i}^{s}\right)$ to denote the equilibrium profit of firm $i$ upon entry of $B 1, B 2$ under SS (under JS).

Lemma 4 (HJM) In game $\Gamma^{s}$, suppose that firms B1 and B2 have entered. Then

(1) Under SS, the equilibrium prices and profits for firms A and B1, B2 are:

$$
\begin{aligned}
& p_{A 1}^{s}=p_{A 2}^{s}=p_{A}^{*}, \quad p_{B 1}^{s}=p_{B 2}^{s}=p_{B}^{*} \quad \text { from (5) } \\
& \pi_{A}^{s}=\frac{(3 t+\alpha)^{2}}{9 t}=\pi_{A}^{g}, \quad \pi_{B 1}^{s}=\pi_{B 2}^{s}=\hat{\pi}
\end{aligned}
$$

(2) Under JS, the equilibrium prices and profits for firms A and B1, B2 are:

$$
\begin{aligned}
P_{A}^{s} & =\frac{1}{5}\left(6 \alpha-6 t+4 \beta^{s}\right), \quad P_{B 1}^{s}=P_{B 2}^{s}=\frac{1}{5}\left(t-\alpha+\beta^{s}\right) \quad \text { with } \beta^{s}=\sqrt{(\alpha-t)^{2}+10 t^{2}} \\
\Pi_{A}^{s} & =\frac{2\left(19 t^{2}-\alpha^{2}+2 \alpha t+(\alpha-t) \beta^{s}\right)^{2}}{125\left(t-\alpha+\beta^{s}\right) t^{2}}, \quad \Pi_{B 1}^{s}=\Pi_{B 2}^{s}=\frac{\left(t-\alpha+\beta^{s}\right)^{3}}{250 t^{2}}
\end{aligned}
$$

(3) If $\alpha<\alpha_{A}^{s}$, then $\pi_{A}^{s}>\Pi_{A}^{s}$; if $\alpha>\alpha_{A}^{s}$, then $\pi_{A}^{s}<\Pi_{A}^{s}$, with $\alpha_{A}^{s}=0.307 t$.

(4) If $\alpha<\alpha_{B 12}^{s}$, then $\hat{\pi}>\Pi_{B 1}^{s}$; if $\alpha>\alpha_{B 12}^{s}$, then $\hat{\pi}<\Pi_{B 1}^{s}$, with $\alpha_{B 12}^{s}=2.092 t$.

For the analysis of $\Gamma^{s}$, Lemma 4(3) is a key result, analogous to Lemma 1(3) for $\Gamma^{g}$, as it determines the choice of firm $A$ at stage two between SS and JS, upon entry of $B 1, B 2$. The main difference between Lemma 1(3) and Lemma 4(3) is that in $\Gamma^{s}$ it is more frequent that firm $A$ 's profit under JS is higher than under SS: The inequality $\Pi_{A}^{g}>\pi_{A}^{g}$ holds for $\alpha>\alpha_{A}^{g}$, whereas $\Pi_{A}^{s}>\pi_{A}^{s}$ holds for $\alpha>\alpha_{A}^{s}$, and $\alpha_{A}^{s}<\alpha_{A}^{g}$. Since $\Gamma^{g}$ and $\Gamma^{s}$ are equivalent under SS, we compare the two games under JS. In this case the Cournot complement effect applies and determines a difference between $\Gamma^{g}$ and $\Gamma^{s}$.

Under JS, in $\Gamma^{s}$ firms $B 1, B 2$ charge a higher total price than firm $B$ in $\Gamma^{g}$; this increases $A$ 's demand and profit:

$$
\Pi_{A}^{S}>\Pi_{A}^{g} \quad \text { for each } \alpha
$$

Moreover, $B 1, B 2$ are collectively better off in $\Gamma^{s}$ than is firm $B$ in $\Gamma^{g}$ : $\Pi_{B 1}^{s}+\Pi_{B 2}^{s}>\Pi_{B}^{g}$, or equivalently, since $\Pi_{B 1}^{s}=\Pi_{B 2}^{s}$,

$$
\Pi_{B 1}^{s}>\frac{1}{2} \Pi_{B}^{g} \quad \text { for each } \alpha
$$


The reason is that since $P_{B 1}^{s}+P_{B 2}^{s}$ is higher than $P_{B}^{g}$, it is profitable for firm $A$ to increase $P_{A}$, as prices are strategic complements, and indeed $P_{A}^{s}>P_{A}^{g}$. This benefits firms $B 1, B 2$ as it increases the demand for bundle $B 1 \& B 2$. Hence $B 1, B 2$ are hurt by their lack of coordination: for each given $P_{A}$, their total profit is lower than the profit that an integrated firm $B$ would earn. But $B 1, B 2$ benefit from the fact that $A$ charges a higher $P_{A}$ than against an integrated firm $B$. We find that the latter effect dominates the former and implies (9). This explains why the inequality $\hat{\pi}<\Pi_{B}^{g} / 2$ holds for $\alpha>\alpha_{B}^{g}$; but $\hat{\pi}<\Pi_{B 1}^{s}$ holds more frequently, for $\alpha>\alpha_{B 12}^{s}$. Therefore, for each firm competition under JS is more profitable in $\Gamma^{s}$ than in $\Gamma^{g}$.

\subsubsection{Subgames Such That Only One Specialist Firm Has Entered}

Suppose that only one specialist firm has entered: say, $B 1$. If $A$ has chosen SS at stage two, then $A$ faces competition in market 1 but is a monopolist in market 2 . Then the equilibrium prices are described by Lemma 1(1) for market 1 , and by Lemma 2(1) for market 2.

Matters are more complicated if firm $A$ bundles because $A$ 's bundle competes with the single product $B 1$ and each consumer chooses one of these alternatives. If the consumer buys product $B 1$, then he does not consume any good 2 (and a social welfare loss occurs). Therefore, this game differs substantially from the pricing games considered above, as $A$ 's bundle includes product $A 2$ but no competitor of $A$ offers product $B 2$.

Nevertheless, the purchase choice of each consumer is readily determined: If the consumer is located in $\left(x_{1}, x_{2}\right)$, then he buys the bundle of $A$ if and only if $2 v+2 \alpha-t x_{1}-t x_{2}-P_{A} \geq v-t\left(1-x_{1}\right)-P_{B 1}$, which reduces to $x_{2} \leq-2 x_{1}+\left(v+2 \alpha+t+P_{B 1}-P_{A}\right) / t$. Therefore the demand for $A$ 's bundle is equal to 1 if $P_{A}-P_{B 1}<v+2 \alpha-2 t$; is 0 if $P_{A}-P_{B 1}>v+2 \alpha+t$; and if $v+2 \alpha-2 t \leq P_{A}-P_{B 1} \leq v+2 \alpha+t$ it is

$$
D_{A}\left(P_{A}, P_{B 1}\right)=\left\{\begin{array}{cc}
1-\frac{1}{42^{2}}\left(2 t-v-2 \alpha-P_{B 1}+P_{A}\right)^{2} & \text { if } v+2 \alpha-2 t \leq P_{A}-P_{B 1} \leq v+2 \alpha-t \\
\frac{1}{4 t}\left(2 v+4 \alpha+t+2 P_{B 1}-2 P_{A}\right) & \text { if } v+2 \alpha-t<P_{A}-P_{B 1} \leq v+2 \alpha \\
\frac{1}{4 t^{2}}\left(v+2 \alpha+t+P_{B 1}-P_{A}\right)^{2} & \text { if } v+2 \alpha<P_{A}-P_{B 1} \leq v+2 \alpha+t
\end{array}\right.
$$

The demand for product $B 1$ is $1-D_{A}\left(P_{A}, P_{B 1}\right)$, and the profit functions are $P_{A} D_{A}\left(P_{A}, P_{B 1}\right), P_{B 1}\left[1-D_{A}\left(P_{A}, P_{B 1}\right)\right]$ for $A$ and for $B 1$, respectively. We use $\pi_{A}^{s 1}, \pi_{B 1}^{s 1}$ $\left(\Pi_{A}^{s 1}, \Pi_{B 1}^{s 1}\right)$ to denote the equilibrium profits under SS (under JS) when only $B 1$ enters.

Lemma 5 In game $\Gamma^{s}$, suppose that only firm $B 1$ has entered. Then

(1) Under SS, the equilibrium prices and profits for firms $A$ and $B 1$ are

14 The analysis of this pricing game when $\alpha=0$ appears in a previous version of HJM. 


$$
\begin{array}{lrl}
p_{A 1}^{s 1}=p_{A}^{*}, & p_{A 2}^{s 1}=v+\alpha-t, & p_{B 1}^{s 1}=p_{B}^{*} \\
\pi_{A}^{s 1}=\frac{1}{18 t}(3 t+\alpha)^{2}+v+\alpha-t, & \pi_{B 1}^{s 1}=\hat{\pi}
\end{array}
$$

(2) Under JS, the equilibrium prices and profits for firms A and B1 are

$$
\begin{aligned}
& P_{A}^{s 1}=\frac{5}{8}(v+2 \alpha)-\frac{5}{4} t+\frac{3}{8} \beta^{s 1}, \quad P_{B 1}^{s 1}=\frac{1}{8} \beta^{s 1}-\frac{1}{8}(v+2 \alpha)+\frac{1}{4} t \quad \text { with } \beta^{s 1}=\sqrt{(v+2 \alpha-2 t)^{2}+16 t^{2}} \\
& \Pi_{A}^{s 1}=P_{A}^{s 1} D_{A}\left(P_{A}^{s 1}, P_{B 1}^{s 1}\right), \quad \Pi_{B 1}^{s 1}=P_{B 1}^{s 1}\left[1-D_{A}\left(P_{A}^{s 1}, P_{B 1}^{s 1}\right)\right]
\end{aligned}
$$

(3) For each $v \geq 3$ t, there exist $\alpha_{A}^{s 1}(v), \alpha_{B 1}^{s 1}(v)$ such that if $\alpha<\alpha_{A}^{s 1}(v)$, then $\pi_{A}^{s 1}>\Pi_{A}^{s 1}$, if $\alpha>\alpha_{A}^{s 1}(v)$, then $\pi_{A}^{s 1}<\Pi_{A}^{s 1}$; if $\alpha<\alpha_{B 1}^{s 1}(v)$, then $\pi_{B 1}^{s 1}>\Pi_{B 1}^{s 1}$; if $\alpha>\alpha_{B 1}^{s 1}(v)$, then $\pi_{B 1}^{s 1}<\Pi_{B 1}^{s 1}$.

Moreover, $\alpha_{A}^{s 1}, \alpha_{B 1}^{s 1}$ are increasing, and $\alpha_{A}^{s 1}(3 t)=2.1 t, \alpha_{B 1}^{s 1}(3 t)=2.776 t$.

The results in Lemma 5(3) can be explained using the demand size effect and the demand elasticity effect as in Sect. 3.1.1: Moving from SS to JS with $\left(P_{A}, P_{B 1}\right)=\left(p_{A 1}^{s 1}+p_{A 2}^{s 1}, p_{B 1}^{s 1}\right)$ makes firm $A$ increase its market share in market 1 , but $A$ loses market share in market 2 because (1) under SS all consumers buy A2; (2) under JS a few consumers buy product $B 1$ and no good 2 at all. Therefore the net effect on $A$ 's profit is ambiguous: The profit loss in market 2 may not be compensated by the profit increase in market 1 . In fact, $A$ 's total profit increases if $\alpha$ is large (or $v$ is not too large), as then $A$ 's market share (and profit) loss in market 2 is small. The demand size effect reduces firm $B 1$ 's profit since the sales of its single product decrease.

In addition, the reduced market share for $B 1$ makes the demand for product $B 1$ elastic, even though a small decrease in $P_{B 1}$ at $\left(P_{A}, P_{B 1}\right)=\left(p_{A 1}^{s 1}+p_{A 2}^{s 1}, p_{B 1}^{s 1}\right)$ allows $B 1$ to win over relatively few consumers (this principle applies also in Sect. 3.1.1 to firm $B$ ). Thus the demand elasticity effect induces firm $B 1$ to reduce $P_{B 1}$ below $p_{B 1}^{s 1}$. This harms firm $A$ and makes $\Pi_{A}^{s 1}$ smaller than $\pi_{A}^{s 1}$ unless $\alpha$ is larger than $\alpha_{A}^{s 1}(v)$ in Lemma 5(3). Finally, we notice that for $\alpha$ large - close to 3t-the demand for $A$ 's bundle is rigid (as in the games of competition under JS examined above), which induces $A$ to increase $P_{A}$ above $p_{A 1}^{s 1}+p_{A 2}^{s 1}$. This benefits firm $B 1$ and makes $\Pi_{B 1}^{s 1}$ greater than $\pi_{B 1}^{s 1}$

\subsubsection{Subgames Such That Neither B1 Nor B2 Has Entered}

In this case $A$ is a monopolist, as in $\Gamma^{g}$ when $B$ does not enter. Hence Lemma 2 applies.

\subsection{Stage Two in $\Gamma^{s}$}

At stage two, firm $A$ chooses between SS and JS by comparing its profit under two alternatives, as in $\Gamma^{g}$ (see Sect. 3.2 and Lemma 3). However, now matters are more 
complicated than in $\Gamma^{g}$ because there are three possible cases for the specialists' entry decisions: both specialists enter; only one enters; no specialist enters. Lemmas 2, 4(3), 5(3) lead to the following results:

Lemma 6 In game $\Gamma^{s}$, at stage two:

(1) if B1 and B2 have entered, then A's best action is SS if $\alpha<\alpha_{A}^{s}$, and is JS if $\alpha>\alpha_{A}^{s}$;

(2) if only one specialist has entered, then A's best action depends on $\alpha$ and $v$ : for each $v \geq 3 t$, A plays SS if $\alpha<\alpha_{A}^{s 1}(v)$, plays JS if $\alpha>\alpha_{A}^{s 1}(v)$;

(3) if no specialist has entered, then A's best action is JS.

\subsection{Stage One in $\Gamma^{s}$}

Here we examine the entry decisions of $B 1, B 2$ at stage one. In $\Gamma^{g}$, the entry decision of firm $B$ is straightforward given Lemmas 1,3 because $B$ is the sole entrant. But in $\Gamma^{s}$ there is strategic interdependence between $B 1$ and $B 2$ : The profitability of entry for $B 1$ may depend on whether $B 2$ enters or not because that affects $A$ 's reaction at stage two (and also price competition at stage three). Therefore we examine an entry game between $B 1$ and $B 2$ in which the payoffs depend on the actions of $B 1, B 2$ and take into account the anticipated choice of $A$ at stage two, which is described by Lemma 6. By Lemma 6(1), we distinguish below the case of $\alpha<\alpha_{A}^{s}$ from the case of $\alpha>\alpha_{A^{*}}{ }^{15}$

For each specialist the gross profit from entering is $\hat{\pi}$, or $\Pi_{B 1}^{s}$, or $\Pi_{B 1}^{s 1}$ (with $\Pi_{B 1}^{s 1}<\Pi_{B 1}^{s}$ ), and since the entry cost is $k$ we can conclude that no specialist enters if $k>\max \left\{\hat{\pi}, \Pi_{B 1}^{s}\right\}$.

Case of $\alpha<\alpha_{A}^{s}$ In this case $A$ chooses SS if at least one specialist has entered. Hence, the game at stage one between $B 1$ and $B 2$ has the following normal form, in which the action In means entry, the action Out means no entry:

\begin{tabular}{|ccc|}
\hline$B 1 \backslash B 2$ & In & Out \\
In & $\hat{\pi}-k, \hat{\pi}-k$ & $\hat{\pi}-k, 0$ \\
Out & $0, \hat{\pi}-k$ & 0,0 \\
\hline
\end{tabular}

In this game the profit of $B 1$ and of $B 2$ is independent of the action of the other firm, hence there is no strategic interdependence between $B 1$ and $B 2$ in (10). This occurs because each specialist that enters competes with $A$ under SS; and in the event that both enter there is no link between market 1 and market 2; hence for a specialist it does not matter whether the other specialist enters or not. Then for each firm In is strictly dominant if $k<\hat{\pi}$; in such case $(I n, I n)$ is the unique Nash Equilibrium (NE

\footnotetext{
${ }^{15}$ By Lemma 6(2), we may also distinguish between $\alpha<\alpha_{A}^{s 1}(v)$ and $\alpha>\alpha_{A}^{s 1}(v)$ for the case in which only one specialist enters. But we show below that this is basically irrelevant from the point of view of specialists' entry.
} 
from now on) of (10). Conversely, Out is strictly dominant if $k>\hat{\pi}$. Next proposition summarizes the analysis of stage one when $\alpha<\alpha_{A}^{s}{ }^{16}$

Proposition 2a In game $\Gamma^{s}$, suppose that $\alpha<\alpha_{A}^{s}$. Then the unique SPNE of $\Gamma^{s}$ is such that firms $B 1$ and $B 2$ both enter if $k<\hat{\pi}$, and neither $B 1$ nor B2 enters if $\hat{\pi}<k$.

We can summarize Proposition 2a by noticing that given $\alpha<\alpha_{A}^{s}$, any entry induces $A$ to play SS. Hence each entrant's net profit is $\hat{\pi}-k$, and entry takes place if and only if $k<\hat{\pi}$.

Case of $\alpha>\alpha_{A}^{s}$ When $\alpha_{A}^{s}<\alpha$, firm $A$ chooses JS if $B 1, B 2$ enter; if only one specialist enters, then $A$ plays SS if $\alpha<\alpha_{A}^{s 1}(v)$, plays JS if $\alpha>\alpha_{A}^{s 1}(v)$. Therefore we distinguish between these cases, starting with $\alpha<\alpha_{A}^{s 1}(v)$ and recall that $\alpha_{A}^{s 1}(v) \geq \alpha_{A}^{s 1}(3 t)=2.1 t$. Then the game at stage one between $B 1$ and $B 2$ is described by (11) below.

For (11), we distinguish two subcases: The first one is such that $\Pi_{B 1}^{s}<\hat{\pi}$ : $\alpha \in\left(\alpha_{A}^{s}, \alpha_{B 12}^{s}\right)$; the second one is such that $\Pi_{B 1}^{s}>\hat{\pi}: \alpha \in\left(\alpha_{B 12}^{s}, \alpha_{A}^{s 1}(v)\right)$. This distinction is important because when $\Pi_{B 1}^{s}<\hat{\pi}$, from (11) we see that a specialist firm that enters-say, $B 1$ - is better off if $B 2$ does not enter: entry by $B 2$ induces $A$ to choose JS, which hurts $B 1$ with respect to SS. Conversely, if $\Pi_{B 1}^{s}>\hat{\pi}$, then an entrant $B 1$ prefers that also $B 2$ enters because $B 1$ is better off if $A$ chooses JS. These differences affect the structure of the NE of (11).

\begin{tabular}{|ccc|}
\hline$B 1 \backslash B 2$ & In & Out \\
In & $\Pi_{B 1}^{s}-k, \Pi_{B 1}^{s}-k$ & $\hat{\pi}-k, 0$ \\
Out & $0, \hat{\pi}-k$ & 0,0 \\
\hline
\end{tabular}

Subcase 1 When $\Pi_{B 1}^{s}<\hat{\pi}$ (i.e., $\alpha<\alpha_{B 12}^{s}$ ) if $k<\Pi_{B 1}^{s}<\hat{\pi}$, then $I n$ is strictly dominant in (11) for each firm, and (In, In) is the unique NE. If instead $\Pi_{B 1}^{s}<k<\hat{\pi}$, then (11) is a hawk-dove game with two asymmetric NE, (Out, In) and (In, Out), and the following symmetric mixed strategy NE (in which each specialist's profit is zero):

$$
\text { firm } B 1(B 2) \text { enters with probability } \frac{\hat{\pi}-k}{\hat{\pi}-\Pi_{B 1}^{s}} \text {, and stays out with probability } \frac{k-\Pi_{B 1}^{s}}{\hat{\pi}-\Pi_{B 1}^{s}}
$$

Since (11) is a symmetric game and (12) is its unique symmetric NE, we assume that $B 1, B 2$ play (12) when $\Pi_{B 1}^{s}<k<\hat{\pi}$.

Subcase 2 When $\hat{\pi}<\Pi_{B 1}^{s}$ (i.e., $\alpha>\alpha_{B 12}^{s}$ ), if $k<\hat{\pi}<\Pi_{B 1}^{s}$ then again $I n$ is strictly dominant for each firm and (In, In) is the unique NE of (11). But if $\hat{\pi}<k<\Pi_{B 1}^{s}$, then (11) is a coordination game with three NE: (In, In), (Out, Out), and the mixed strategy NE (12). All of the NE are symmetric; hence we use Pareto dominance as a refinement. Since $(I n, I n)$ Pareto dominates the other NE, we suppose that $B 1, B 2$ play (In, In) when $\hat{\pi}<k<\Pi_{B 1}^{s}$.

\footnotetext{
${ }^{16}$ The rest of the SPNE strategies for $\Gamma^{s}$, relative to stages two and three, is obtained from Lemmas 2, 4-6.
} 
The above analysis holds as long as $\alpha<\alpha_{A}^{s 1}(v)$. If conversely $\alpha>\alpha_{A}^{s 1}(v)$, then $\alpha>2.1 t>\alpha_{B 12}^{s}$; hence $\hat{\pi}<\Pi_{B 1}^{s}$ as in Subcase 2. Then firm $A$ plays JS if only one specialist enters, and the specialist's gross profit is $\Pi_{B 1}^{s 1}$ (from Lemma 5(2)). Therefore $\hat{\pi}$ in (11) must be replaced by $\Pi_{B 1}^{s 1}$, and $\Pi_{B 1}^{s 1}<\Pi_{B 1}^{s}$ for each $\alpha>\alpha_{A}^{s 1}(v)$. Hence the arguments of Subcase 2 still apply to establish that $(I n, I n)$ is either the unique NE or the unique Pareto dominant NE of (11).

Next proposition summarizes the analysis of stage one for $\alpha>\alpha_{A}^{s}$ (Footnote 16 still applies):

Proposition $\mathbf{2 b}$ In game $\Gamma^{s}$, suppose that $\alpha>\alpha_{A^{*}}^{s}$. Then neither B1 nor B2 enters if $k>\max \left\{\hat{\pi}, \Pi_{B 1}^{s}\right\}$. If instead $k<\max \left\{\hat{\pi}, \Pi_{B 1}^{s}\right\}$, then the stage one entry game is such that:

(1) for $\alpha \in\left(\alpha_{A}^{s}, \alpha_{B 12}^{s}\right)$, (In, In) is the unique $N E$ if $k<\Pi_{B 1}^{s}$; the unique symmetric $N E$ is (12) if $\Pi_{B 1}^{s}<k<\hat{\pi}$;

(2) for $\alpha>\alpha_{B 12}^{S}$ and for each $k<\Pi_{B 1}^{s}$, (In, In) is either the unique NE or the unique Pareto dominant NE.

The message of Proposition 2b is that if $\alpha \in\left(\alpha_{A}^{s}, \alpha_{B 12}^{s}\right)$, then $A$ plays JS in case $B 1, B 2$ enter. This reduces the profit of $B 1$ and of $B 2$ from $\hat{\pi}$ to $\Pi_{B 1}^{s}$ and deters entry if $\Pi_{B 1}^{s}<k<\hat{\pi}$ in the sense that each specialist enters with probability less than one (conversely, entry of $B 1, B 2$ occurs with certainty if $k<\Pi_{B 1}^{s}$ ). If instead $\alpha>\alpha_{B 12}^{s}$, then $\Pi_{B 1}^{s}>\hat{\pi}$, and JS actually favors entry as it increases each specialist's profit.

\section{Comparison Between $\Gamma^{g}$ and $\Gamma^{s}$}

In this section we compare the outcomes of $\Gamma^{g}$ and $\Gamma^{s}$ in terms of the firms' profits. ${ }^{17}$ For firms $B$ and $B 1, B 2$ we compare the profit of $B$ in $\Gamma^{g}$ with the sum of the profits of $B 1, B 2$ in $\Gamma^{s}$.

We first notice that $\Gamma^{g}$ and $\Gamma^{s}$ lead to the same outcome if $\alpha<\alpha_{A}^{s}$ or $k>\max \left\{\hat{\pi}, \Pi_{B 1}^{s}\right\}$ : If $\alpha<\alpha_{A}^{s}$ then $A$ chooses SS whenever some entry occurs (Lemmas 3(1), $6(1-2)$ ), both in $\Gamma^{g}$ and in $\Gamma^{s}$. Thus, in both games firm $A$ is a monopolist if $\hat{\pi}<k$, faces competition in both markets (under SS) if $k<\hat{\pi}$, and the same outcome arises. When $k>\max \left\{\hat{\pi}, \Pi_{B 1}^{s}\right\}$, in both $\Gamma^{g}$ and $\Gamma^{s}$ the high entry cost deters any entry, and $A$ is a monopolist.

Therefore, in the following we consider $\alpha>\alpha_{A}^{s}$ and $k<\max \left\{\hat{\pi}, \Pi_{B 1}^{s}\right\}$, and recall that Lemmas 1,4 show that

$$
\alpha_{A}^{s}<\alpha_{A}^{g}<\alpha_{B 12}^{s}<\alpha_{B}^{g} \quad \text { and } \quad \max \left\{\hat{\pi}, \Pi_{B 1}^{s}\right\}=\left\{\begin{array}{cc}
\hat{\pi} & \text { if } \alpha \in\left(\alpha_{A}^{s}, \alpha_{B 12}^{s}\right) \\
\Pi_{B 1}^{s} & \text { if } \alpha>\alpha_{B 12}^{s}
\end{array}\right.
$$

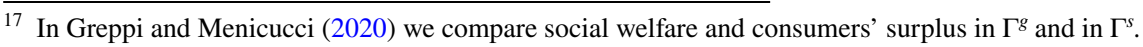




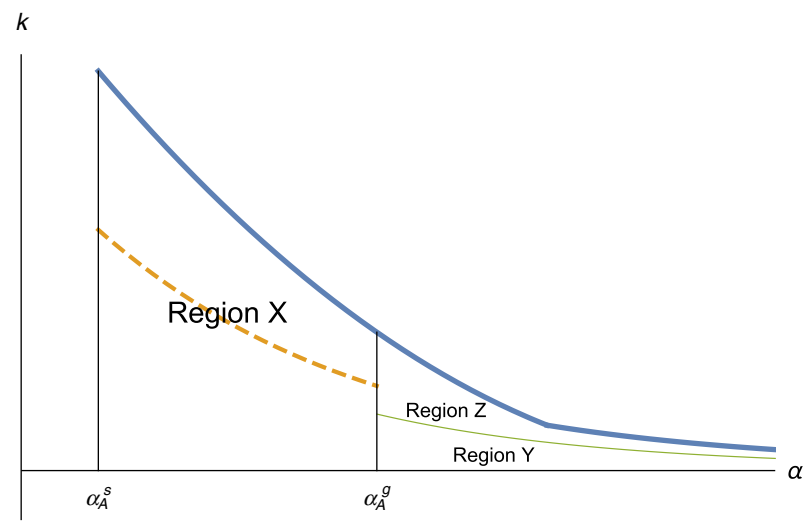

Fig. $2 c k=\max \left\{\hat{\pi}, \Pi_{B 1}^{s}\right\}$ (thick), $k=\Pi_{B 1}^{s}$ (dashed), $k=\Pi_{B}^{g} / 2$ (thin)

In $\Gamma^{s}$, for each $\alpha>\alpha_{A}^{s}$ firm $A$ plays JS if $B 1, B 2$ enter. Conversely, in $\Gamma^{g}$ firm $A^{\prime}$ 's choice upon entry of $B$ depends on $\alpha$ : $A$ plays SS if $\alpha<\alpha_{A}^{g}$, plays JS if $\alpha>\alpha_{A}^{g}$. Therefore we distinguish $\alpha \in\left(\alpha_{A}^{s}, \alpha_{A}^{g}\right)$ from $\alpha>\alpha_{A}^{g}$. To this purpose we use Fig. 2 above, in the space $\alpha, k$, in which the thick curve is the graph of $k=\max \left\{\hat{\pi}, \Pi_{B 1}^{s}\right\}$. Region $X$ consists of the points such that $\alpha \in\left(\alpha_{A}^{s}, \alpha_{A}^{g}\right)$ and $k<\max \left\{\hat{\pi}, \Pi_{B 1}^{s}\right\}$. The dashed curve in Region $X$ is the graph of $k=\Pi_{B 1}^{s}$; by (13), it lies below the thick curve. The points $(\alpha, k)$ such that $\alpha>\alpha_{A}^{g}$ and $k<\max \left\{\hat{\pi}, \Pi_{B 1}^{s}\right\}$ belong to Region $Y$ or $Z$. The solid thin curve separating the two regions is the graph of $k=\Pi_{B}^{g} / 2$; it lies below the thick curve since $\Pi_{B}^{g} / 2<\Pi_{B 1}^{s}$ by (9).

\subsection{Case of $\alpha \in\left(\alpha_{A^{\prime}}^{s}, \alpha_{A}^{g}\right)($ Region $X$ in Fig. 2)}

In Region $X$ we first consider $k$ smaller than $\Pi_{B 1}^{s}:(\alpha, k)$ below the dashed curve. Then in $\Gamma^{s}$ firms $B 1, B 2$ enter, $A$ plays JS, and $A(B 1, B 2)$ earns $\Pi_{A}^{s}\left(\right.$ earns $\left.\Pi_{B 1}^{s}-k\right)$. In $\Gamma^{g}$, firm $B$ enters as $k<\Pi_{B 1}^{s}<\hat{\pi}, A$ plays SS, and $A(B)$ earns $\pi_{A}^{g}$ (earns $2 \hat{\pi}-2 k$ ). Hence, $A$ prefers $\Gamma^{s}$ since competition under JS is softer in $\Gamma^{s}$ than in $\Gamma^{g}$ (see (8)), which makes JS more profitable for $A$ than SS. Conversely, $B$ prefers $\Gamma^{g}$ as JS in $\Gamma^{s}$ reduces the profit of $B 1, B 2: \Pi_{B 1}^{s}<\hat{\pi}$.

The fact that JS in $\Gamma^{s}$ reduces the profit of $B 1, B 2$ is especially relevant if $k$ is greater than $\Pi_{B 1}^{s}$ but smaller than $\hat{\pi}-(\alpha, k)$ in Region $X$ is between the dashed and the thick curve-because then entry in $\Gamma^{s}$ is affected: For $B 1, B 2$ it is unprofitable to enter jointly (see (11)), and by Proposition $2 b(1)$ each specialist enters with probability less than one, according to (12).

It follows that firm $A$ is better off in $\Gamma^{s}$ than in $\Gamma^{g}$ in the whole Region $X$ : $A$ would play SS in $\Gamma^{g}$, but in $\Gamma^{s}$, (1) if $k<\Pi_{B 1}^{s}$, then $B 1, B 2$ enter and $A$ prefers JS to SS; 
(2) if $k>\Pi_{B 1}^{s}$, then entry of $B 1, B 2$ is deterred with positive probability, hence $A$ becomes a monopolist in one market, or both, with positive probability, which is more profitable than facing competition.

The root of the result is that in $\Gamma^{S}$ competition under JS is more profitable for $A$ than under SS if entry occurs, and it reduces the profits of $B 1, B 2$. Hence, $A$ 's ability to bundle creates in $\Gamma^{s}$ an entry barrier against $B 1, B 2$ for some values of $k$. This is not the case for $\Gamma^{g}$ because bundling reduces $B$ 's profit, but reduces also $A$ 's profit given $B$ 's entry when $\alpha<\alpha_{A}^{g}$; hence $B$ profitably enters as long as $k<\hat{\pi}$ (Proposition 1(1)). Here the fact that competition under JS is tougher in $\Gamma^{g}$ than in $\Gamma^{S}$ is useful for $B$ as it induces $A$ to choose SS.

Case of $\alpha>\alpha_{A}^{g}$ (Regions $Y, Z$ in Fig. 2) For $(\alpha, k)$ in Regions $Y$ or $Z$, in $\Gamma^{g}$ firm $A$ chooses JS after $B$ 's entry; hence $B$ enters if and only if $k<\Pi_{B}^{g} / 2$, that is if $(\alpha, k)$ is in Region $Y$. In $\Gamma^{s}$, for $(\alpha, k)$ in Region $Y$, firms $B 1, B 2$ enter since $k<\Pi_{B 1}^{s}$ holds. Therefore, the comparison between $\Gamma^{g}$ and $\Gamma^{s}$ in Region $Y$ reduces to the comparison under JS of competition between $A$ and $B$ or among $A, B 1, B 2$. Then (8), (9) reveal that $A$ and $B$ both prefer $\Gamma^{s}$.

Finally, for $(\alpha, k)$ in Region $Z$, in $\Gamma^{g}$ firm $B$ stays out because its gross profit from entry $\Pi_{B}^{g}$ is smaller than its entry cost $2 k$; thus $\Gamma^{g}$ leads to $A$ 's monopoly. Conversely, in $\Gamma^{s}$ both $B 1$ and $B 2$ enter with positive probability (actually, they enter with certainty if $k<\Pi_{B 1}^{s}$ ), thus $A$ is less often a monopolist in $\Gamma^{s}$ than in $\Gamma^{g}$; this makes $A$ prefer $\Gamma^{g}$ and $B$ prefer $\Gamma^{s}$.

The difference with respect to Region $X$ is that when $\alpha>\alpha_{A}^{g}$, JS is profitable for $A$ in $\Gamma^{g}$; thus $B$ enters if $(\alpha, k)$ is in Region $Y$, does not enter if $(\alpha, k)$ is in Region $Z$. But in $\Gamma^{s}$ both $B 1, B 2$ enter with positive probability if $(\alpha, k)$ is in Region $Z$. Therefore in this region $A$ 's ability to bundle is a more effective foreclosure instrument in $\Gamma^{g}$ than in $\Gamma^{s} \cdot{ }^{18}$ Unlike in Region $X$, here the fact that competition under JS is tougher in $\Gamma^{g}$ than in $\Gamma^{s}$ is a disadvantage for $B$ as it makes its entry more difficult.

The next proposition summarizes the three cases we have considered:

Proposition 3 Suppose that $\alpha>\alpha_{A}^{s}$ and $k<\max \left\{\hat{\pi}, \Pi_{B 1}^{s}\right\}$, otherwise $\Gamma^{g}$ and $\Gamma^{s}$ lead to the same outcome. Then:

(1) A prefers $\Gamma^{s}, B$ prefers $\Gamma^{g}$ if $\alpha \in\left(\alpha_{A}^{s}, \alpha_{A}^{g}\right)$ (Region $X$ in Fig. 2);

(2) A, B both prefer $\Gamma^{s}$ if $\alpha>\alpha_{A}^{g}$ and $k<\Pi_{B}^{g} / 2$ (Region Y in Fig. 2);

(3) A prefers $\Gamma^{g}, B$ prefers $\Gamma^{s}$ if $\alpha>\alpha_{A}^{g}$ and $\Pi_{B}^{g} / 2<k$ (Region $Z$ in Fig. 2).

\section{Conclusions}

This paper examines the effects of the ability to bundle of a firm that offers two higher-quality products and faces an entry threat from an integrated rival or from separate rivals. One significant extension, left to future research, would allow for more than two products, product-specific quality differences, and the possibility of mergers among a few, but not all, specialist firms.

\footnotetext{
18 This claim applies if $\alpha<\alpha_{B 12}^{s}$, because for $\alpha>\alpha_{B 12}^{s}$, JS actually favors entry in $\Gamma^{s}$ (and in $\Gamma^{g}$ if $\alpha>\alpha_{B}^{g}$ ) with respect to SS, but entry remains more difficult in $\Gamma^{g}$ than in $\Gamma^{s}$.
} 
Acknowledgements We are grateful to two anonymous referees and (especially) to the Editor of this journal for various comments, which helped us to improve the paper.

Funding Open access funding provided by Università degli Studi di Firenze within the CRUI-CARE Agreement.

\section{Compliance with Ethical Standards}

Conflict of interest The authors declare that they have no conflict of interest.

Open Access This article is licensed under a Creative Commons Attribution 4.0 International License, which permits use, sharing, adaptation, distribution and reproduction in any medium or format, as long as you give appropriate credit to the original author(s) and the source, provide a link to the Creative Commons licence, and indicate if changes were made. The images or other third party material in this article are included in the article's Creative Commons licence, unless indicated otherwise in a credit line to the material. If material is not included in the article's Creative Commons licence and your intended use is not permitted by statutory regulation or exceeds the permitted use, you will need to obtain permission directly from the copyright holder. To view a copy of this licence, visit http://creativecommons.org/licen ses/by/4.0/.

\section{References}

Armstrong, M. (1996). Multiproduct nonlinear pricing. Econometrica, 64(1), 51-75.

Carlton, D. W., \& Waldman, M. (2002). The strategic use of tying to preserve and create market power in evolving industries. RAND Journal of Economics, 33(2), 194-220.

Choi, J. P. (2008). Mergers with bundling in complementary markets. Journal of Industrial Economics, 56(3), 553-577.

Choi, J. P., \& Stefanadis, C. (2001). Tying, investment, and the dynamic leverage theory. RAND Journal of Economics, 32(1), 52-71.

Denicolò, V. (2000). Compatibility and bundling with generalist and specialist firms. Journal of Industrial Economics, 48(2), 177-188.

Greppi, A., \& Menicucci, D. (2020). On Bundling and Entry Deterrence, Disei Working Paper, available at https://www.disei.unifi.it/cmpro-v-p-95.html. Accessed 11 Aug 2020.

Hurkens, S., Jeon, D.-S., \& Menicucci, D. (2019). Dominance and competitive bundling. American Economic Journal: Microeconomics, 11(3), 1-33.

Nalebuff, B. (2000). Competing against bundles. In P. J. Hammond \& G. D. Myles (Eds.), Incentives, organization, public economics (pp. 323-336). London: Oxford University Press.

Nalebuff, B. (2002). Bundling and the GE-Honeywell Merger. Yale School of Management Working Paper \#22, available at https://ssrn.com/abstract=327380. Accessed 11 Aug 2020.

Nalebuff, B. (2004). Bundling as an entry barrier. Quarterly Journal of Economics, 119(1), 159-187.

Peitz, M. (2008). Bundling may blockade entry. International Journal of Industrial Organization, 26(1), 41-58.

Whinston, M. (1990). Tying, foreclosure and exclusion. American Economic Review, 80(4), 837-859.

Publisher's Note Springer Nature remains neutral with regard to jurisdictional claims in published maps and institutional affiliations. 